\title{
EFFECTS OF 2,4-DICHLOROPHENOXYACETIC ACID ON CERTAIN ALKALOIDAL PLANTS
}

D. E. G. SHEAT, Massey Agricultural College, Palmerston North,

FXPERIMENTS have been carried out to determine whether lupin plants of differing alkaloid content and at various stages of maturity respond differentially to treatment with 2,4-dichlorophenoxyacetic acid, and further whether this compound causes a lowering of total alkaloid content in the leaves of hemlock (Conium maculatum). Results suggest that with lupins there is no variation in response to treatment attributabie to the effects of this compound and that with hemlock this growth regulator does not in itself affect the alkaloid content of the leaves.

Sylwester (1) has suggested that poisonous plants may be rendered harmless to livestock by spraying with mixtures whose active component is the compound 2,4-D, and this suggestion tends to be supported by the observations of farmers and other persons in this country who are concerned with the control of weeds in pastures. Further, it has been reported (2) that there was a differential response to residual 2,4-D in a spray accidentally applied to plants of an F2 generation of bitter $x$ sweet lupin cross. On the basis of these observations it was suggested that an investigation of the effect of 2,4-D on the alkaloid content or certain plants could be profitable. The following experiments were therefore carried out:

(i) Experiments with Lupins: Field Spraying Trials

Young and mature bitter and sweet lupin plants were sprayed with mixtures containing 2,4-D and the responses of these plants were noted. Varieties treated when plants were at an advanced stage of maturity were Sweet Yellow, Sweet Blue, Sweet White, Nonshattering Yellow, Bitter Blue, and Bitter Pink, and all these varieties except for Nonshattering Yellow were grown in pots and in boxes and treated when only seedlings. Plants in pots were treated with the sodium salt of 2,4-D at different concentrations when the primary leaves of the great majority of the plants were fully opened. Plants in boxes at a stage when the majority had three nodes fully developed were also sprayed with varying concentrations of an aqueous solution of this salt. Mature plants in the field were sprayed with a commercial preparation of the acid at approximately 500 p.p.m. For the young plants the following concentrations of the sodium salt were applied: 10,$000 ; 1000 ; 500 ; 100 ; 50 ; 10 ; 1.0$, and 0.1 p.p.m. It was not possible to find any morphological differences in response which could be due to species, and therefore alkaloid content, variations.

Nevertheless the persistence of the reports that 2,4-D renders poisonous plants palatable to stock could not be denied and an investigation of this assertion seemed warranted. Hemlock, because it was most readily available for experimental purposes, was chosen for investigation.

\section{(ii) Experiments with Hemlock: (a) Field Spraying Trials}

Mature hemlock plants at a stage just before flowering were selected in an open field lying to the sun, and groups of two bushes in all possible conditions of shade and shelter from wind were cleared of surrounding foliage. One bush of each pair was treated with an aqueous solution of the sodium salt of 2,4-D at a concentration of 500 p.p.m. This concentration was known to cause characteristic morphological responses but to be non-lethal (3). 
Twenty-four hours after spraying, hemlock plants showed all the characteristic responses due to treatment with 2,4-D. All young shoots and leaves and stems near the immature floral heads were twisted ano distorted. This condition continued during the 8 days of the experiment and distortion was most pronounced by about the fifth day.

\section{(b) Laboratory Investigations}

Sampling: Samples from treated and control plants were collected for chemical analysis every second day for 8 days and therefore included the period during which the plants were recovering from the effects of the growth substance.

At 2-day intervals and at approximately the same time of day leaves were gathered from control and treated plants, every effort being made to select from each plant one each of a mature, an intermediate, and a young leaf. Soft green segments of the leaves, stripped from petioles anct midribs of leaves in bulked samples were immediately used for chemicai analyses.

Dry matter was determined on representative fractions of the tissue prepared for alkaloid estimation by heating at 100 degrees $C$. to constant weight.

\section{Estimation of Alkaloid Content}

Various methods for analysis of total alkaloids of hemlock and of galenic preparations from this plant are given in the literature (4-10) None of the methods proved suitable with hemlock leaves, because of the different nature of the fresh material and its low alkaloid content. Moreover, the volatility of coniine and the liquid characteristics of some o: its compounds make difficult its separation by precipitation methods.

The following method was finally adopted:

\section{(i) Extraction}

Five hundred grams of leaf tissue were macerated in a Waring Blendor with abuut 1 litre of approximately $0.1 \mathrm{~N} \mathrm{HCl}$ and $50 \mathrm{ml}$. ethyl ether and the mixture was allowed to stand for 24 hours. It was then strained through linen and washed several times with dilute hydrochloric acid and finally filtered by suction. The filtrate was washed twice with chloro form to remove most of the chlorophyll and tars, and the acid aqueous extract was warmed to remove the chloroform from solution.

\section{(i) Distillation and Titration}

Twenty-five $\mathrm{ml}$. of extract (representing about 10 grams of the o:iginal leaf tissue) were taken for analysis. This was made strongly akaline with 20 per cent. sodium hydroxide and boiled for 15 minutes in an all-glass still, the distillation being aided by means of a current os purified air which passed through the boiling liquid and condenser ino a 4 per cent. solution of boric acid. The solution and in boric acid was titrated with a standard solution of sulphuric acid, a mired indicator of bromo-cresol-green and methyl red being used. This pncedure gave good results when tested with standard solutions of piperidile and coniine hydrochloride.

The reproducibility of the whole process of extraction and analysis was tesicd by a pharmaceutical preparation of coniine and macerates of hemlod leaves to which known amounts of coniine hydrochloride had beenadled. It was shown that extractions of the acid aqueous extract with solents did not remove volatile bases. Differences between duplicate estmations did not exceed approximately 5 per cent. when analyses were 
carried out on freshly-picked leaves. Wilted herbage, however, contained considerable amounts of volatile bases (presumably derived from breakdown of proteins), and the method was therefore applied only to fresh material.

Results: Results of these experiments are given in Table $\mathbf{I}$.

Table I.

Coniine content of leaves of hemlock plants sprayed with 2,4-dichlorophenoxyacetic acid

Time of Harvesting:

Days after spraying

Volatile alkaloid*

(Calculated as coniine)

p.p million

Control

Control

Control

Treated

Control

Treated

Control

Treated

Control

Treated

p.m. 9 Nov.

4 p.m. 11 Nov.

4 p.m. 11 Nov.

4 p.m. 13 Nov.

4 p.m. 13 Nov.

4 p.m. 15 Nov.

4 p.m. 15 Nov.

4 p.m. 17 Nov.

4 p.m. 17 Nov

(Dry matter basis)

*Calculated as coniine, the major constituent of 5 alkaloids found in hemlock.

These results suggest that 2,4-dichlorophenoxyacetic acid has no effect on alkaloid synthesis in the plant.

That this particular growth substance applied in toxic and nontoxic concentrations does not affect alkaloid synthesis in Datura stramonium has been demonstrated by Tsao and Youngken (11), and Youngken (12) had already shown that 2,4-D absorbed by the stem of this plant has no significant effect upon total alkaloid formation in the leaves. Thougl it is quite impossible to generalise when the subject of discussion involves the synthesis of alkaloids in different plant genera, it does seem that $i$ the most generally accepted view regarding the origin of alkaloids is plants as waste products of nitrogen metabolism as postulated by Robin. scn (13) and recently supported by Chatterjee (14) is correct, and $\mathrm{i}$ this theory may be extended to hemlock, further support is given th: hypothesis by the work of Chaze (15) and of Dragoni-Testi (16), who hav shown that coniine at least of the alkaloids of hemlock is exuded fron the leaves of the plant through the stomata and is lost by volatilisation. The complex phytochemical reactions involved in the synthesis of the hemlock alkaloids could therefore be explained in terms of this conversior within the plant of waste products of volatile compounds which may b: disposed of by this process of volatilisation. Metsapa (17) had demon strated that many plants containing alkaloids lose part of their actie principle on drying and Chaze (loc. cit.) showed that the process if exudation from the stomata occurred most rapidly during the warmir periods of the day. It can be postulated, therefore, that if, as has ben suggested, the compound 2,4-D can render normally poisonous plants harmless to stock, it is the wilting process consequent upon successil killing of the plant by this substance which is the reason for this lowering of the amounts of the poisonous principle normally found in healty plants; but Goris (18) has pointed out that there is a very wide variatin in total alkaloid contents of hemlock plants according to their habitt, and it seems doubtful whether in this country the leaves of the plat can ever be said to be responsible for the poisoning of stock. 
The author is grateful to the University of New Zealand and Messrs. Ivon Watkins Ltd. for a research grant which partly defrayed expenses incurred in this work.

\section{REFERENCES :}

1. Sylwester, E. P. (1949), "Down to Earth," 5-17. (U.S.A.)

2. Allen, F. C., Crop Research Division, Dept. Sci. and Ind. Res., N.Z. (Private Communication.)

3. Pearse, H. L., Commonwealth Bureau of Horticulture and Plantation Crops, Technical Communication No. 20, 1948.

4. Henry, T. A. (1924), The Plant Alkalotds (Churchill, London), 2nd Edn.

5. U.S. Pharmacopoeia 8th Edn.

6. Farr, E. H., and R. Wright (1887-88), Pharm. Journal 18-13 and 511; (1891) ibid 21-857 and 936.

7. Farr, E. H., and R. Wright (1895-96) ibid $1-89$.

8. Madaus, G., and H. Schindler (1938), Arch. Pharm. 276-280.

9. Bamford, F. (1947), Poisons, Their Isolation and Identification, 2nd Edn. (Churchill, London.)

10. Chaze, J., and M. M. Jarnot (1934) Compt. Rendu (Paris) 198-2015. 11. Tsao, D. P. N., and H. W. Youngken (1949) Journ. Amer. Pharm. Assoc.
Sci. Edn. 38 (2)-112.

12. Youngken, H. W. (1948) ibid 37-196.

13. Robinson, R. (1917), Journal Chem. Soc. 111-876, et seq.

14. Chatterjee, R. (1950) Sci. and Culture 16 (2)-58.

15. Chaze, J. (1933), Compt. Rendu (Paris) 197-1148.

16. Dragoni-Testi, G. (1935) Atti. soc. Ital. prog. Sci. 23 ili-130. (Seen in Chemical Abstracts.)

17. Metsapa, H. (1926) Pharmacia No. 5 (seen in abstract)

18. Goris, A. (1914) Role of Alkaloids in Plants (J. Lechevalier, Paris). (Referred to in (15).) 